Meta

Journal des traducteurs

Translators' Journal

\title{
Prolégomènes à une didactique de la traduction professionnelle
}

\section{Marco A. Fiola}

Volume 48, numéro 3, septembre 2003

Traduction et enseignement

Translation and teaching

URI : https://id.erudit.org/iderudit/007594ar

DOI : https://doi.org/10.7202/007594ar

Aller au sommaire du numéro

Éditeur(s)

Les Presses de l'Université de Montréal

ISSN

0026-0452 (imprimé)

1492-1421 (numérique)

Découvrir la revue

Citer cet article

Fiola, M. A. (2003). Prolégomènes à une didactique de la traduction professionnelle. Meta, 48(3), 336-346. https://doi.org/10.7202/007594ar
Résumé de l'article

Lorsqu'il est question de théorie en enseignement de la traduction, on pense tout de suite à la théorie de la traduction, à la place qu'elle occupe dans l'enseignement et à la part de formation qui doit être réservée au contenu de formation théorique. Dans le présent article, il n'est pas question de théorie de la traduction mais plutôt de la manière dont la recherche en éducation pourrait profiter à la formation des traducteurs professionnels, principalement en ce qui a trait à la conception des programmes de formation. L'auteur présente la notion de situation didactique et illustre comment cette perspective pourrait alimenter la réflexion à l'égard de la didactique de la traduction professionnelle.
Ce document est protégé par la loi sur le droit d'auteur. L'utilisation des services d'Érudit (y compris la reproduction) est assujettie à sa politique d'utilisation que vous pouvez consulter en ligne.

https://apropos.erudit.org/fr/usagers/politique-dutilisation/ 


\title{
Prolégomènes à une didactique de la traduction professionnelle
}

\author{
MARCO A. FIOLA \\ Université du Québec en Outaouais, Gatineau, Canada \\ marco.fiola@uqo.ca
}

\begin{abstract}
RÉSUMÉ
Lorsqu'il est question de théorie en enseignement de la traduction, on pense tout de suite à la théorie de la traduction, à la place qu'elle occupe dans l'enseignement et à la part de formation qui doit être réservée au contenu de formation théorique. Dans le présent article, il n'est pas question de théorie de la traduction mais plutôt de la manière dont la recherche en éducation pourrait profiter à la formation des traducteurs professionnels, principalement en ce qui a trait à la conception des programmes de formation. L'auteur présente la notion de situation didactique et illustre comment cette perspective pourrait alimenter la réflexion à l'égard de la didactique de la traduction professionnelle.
\end{abstract}

\section{ABSTRACT}

When the issues of theory and translator training are raised, what usually comes to mind is whether or not it is relevant to teach translation theory to translation students, and if so, in what proportion to translation practice. This article will focus on theory, but not on translation theory. Instead, the author will demonstrate that drawing on education theory could be beneficial to the training of professional translators, especially with respect to curriculum design. First, a definition of "situation didactique" will be presented, followed by an illustration of how it may benefit the didactics of professional translation.

\section{MOTS-CLÉS/KEYWORDS}

enseignement de la traduction, programmes, didactique, théorie, traduction professionnelle

\section{Introduction}

Au Canada, les programmes d'études ${ }^{1}$ en traduction professionnelle font depuis longtemps l'objet de critiques. Avant 1999, ces critiques viennent tantôt de formateurs (Goffin 1971: 66), tantôt d'apprenants (Horguelin 1975: 42 et passim), tantôt encore d'employeurs (Roberts 1985: 344; Card 1990: 53). Cependant, en 1999, le Rapport du Comité sectoriel de l'industrie canadienne de la traduction ${ }^{2}$ présente une opinion critique et concertée de la formation. Les administrateurs et les concepteurs de programmes d'études doivent maintenant décider de l'opportunité de revoir la composition et la pertinence des programmes actuels à la lumière des critiques contenues dans le Rapport. Partant, il est à propos d'analyser la conjoncture dans une perspective globale à partir de laquelle il sera possible de comprendre l'importance de chacun des éléments dont il faut tenir compte dans la conception et la refonte de ces programmes. 


\section{Traduction, pédagogie et didactique}

La traduction professionnelle est depuis 1936 (Delisle 1987: 65) un sujet d'étude dans les universités canadiennes. Après des débuts modestes, la formation de traducteurs professionnels s'est imposée non seulement comme champ de formation mais également comme champ de recherche. Au Canada, les travaux de Vinay et Darbelnet (1958) et de Delisle (1980) constituent de véritables jalons dans le domaine. Les travaux de ces auteurs visaient à déterminer et à décomposer les éléments de la compétence traductionnelle en vue d'en rationaliser l'apprentissage. À partir d'un concept de «bonne traduction", les auteurs présentent des propositions pédagogiques devant mener à l'acquisition de la compétence traductionnelle, du savoir de métier, du savoirfaire et, dans une certaine mesure, du savoir-être propres au traducteur.

Rappelons que si la pédagogie s'intéresse aux connaissances et aux compétences, elle inclut également les relations en classe devant favoriser l'apprentissage. À l'inverse, la didactique s'attache à une discipline et à son enseignement et cherche à faciliter l'appropriation des connaissances et des compétences (Chaduc 1999: 90). Autrement dit, la pédagogie s'intéresse à ce qui se passe en classe, tandis que la didactique sert à préparer ce qui doit s'y passer. La question de la pédagogie ne sera pas abordée expressément ici. En revanche, il sera question de l'opportunité d'apporter un éclairage didactique, issu des sciences de l'éducation, à la conception et à la refonte des programmes d'enseignement de la traduction. Plus précisément, il sera question de la notion de situation didactique dans le contexte de la formation de traducteurs professionnels.

\section{La situation didactique}

La notion de situation didactique fait référence aux éléments dont il faut tenir compte dans l'enseignement d'une discipline, notamment dans la conception des programmes. Ces programmes englobent certes les mesures pédagogiques mises en œuvre en salle de cours, mais ils comprennent également ce qui intervient, en amont de l'enseignement, ainsi que la finalité des programmes de formation, en aval. Les catégories d'éléments de la situation didactique, au nombre de quatre, sont solidaires et interdépendantes. Ce concept a notamment été mis à profit en traduction pédagogique par Élisabeth Lavault (1998: 25). Il est intéressant de se demander si ce concept s'applique en traduction professionnelle et, le cas échéant, comment en tirer parti.

Bien que le contenu des catégories soit appelé à changer, la situation didactique est composée des quatre catégories suivantes:

a. Les apprenants: comprend notamment la formation préalable, les motivations et les besoins de formation des apprenants;

b. La durée d'apprentissage: englobe la durée des leçons, des cours et des programmes de formation;

c. L'espace et les modalités d'apprentissage: renvoie aux salles de cours, réelles ou virtuelles, au nombre d'apprenants et à l'homogénéité relative du groupe d'apprenants;

d. Les formateurs: inclut leurs intérêts, leur statut professoral, leur formation en pédagogie et leurs motivations propres.

Voici maintenant, une à une, ce qu'englobent ces catégories dans la formation des traducteurs professionnels. 


\subsection{Les apprenants}

Toute situation d'apprentissage mobilise une triade pédagogique composée du formateur, de l'apprenant et du savoir à acquérir. La nature du processus pédagogique mis en œuvre détermine la nature de la relation pédagogique qui unit ces éléments. Voici un bref rappel des trois approches possibles.

D'abord, dans le cas de la performance magistrale, on peut dire que le processus pédagogique est axé sur l'enseignement, car la relation entre le formateur et le savoir est au premier plan et le rôle du formateur est d'exposer le savoir à acquérir; les préoccupations de l'apprenant sont au second plan. Dans cette approche pédagogique standard à l'égard de tous les apprenants, la marge de manœuvre du formateur est très restreinte; son enseignement a d'autant plus de chances de porter ses fruits que la classe est homogène. Partant, il est essentiel d'imposer aux apprenants des critères d'admission qui soient les mieux définis possible, c'est-à-dire qui laissent le moins de place possible aux écarts par rapport au modèle d'apprenant recherché.

Ensuite, on dira que le processus pédagogique est axé sur la formation lorsque la relation d'interaction entre le formateur et l'apprenant est au premier plan et que le savoir passe au second plan. Cette approche est caractérisée par le mode de formation maitre-apprenti, d'apprentissage par l'imitation, ou d'imitation par l'exemple. Cette forme d'encadrement étroit se prête aux situations d'apprentissage par très petits groupes. L'acquisition du savoir est tributaire de la relation entre le formateur et l'apprenant.

Finalement, on dira que le processus pédagogique est axé sur l'apprentissage lorsque la relation entre l'apprenant et le savoir à acquérir est au premier plan et que le formateur est au second plan, occupant une fonction de guide dans le processus d'apprentissage. C'est l'approche socioconstructiviste, approche que préconise Don Kiraly (2000) pour l'enseignement de la traduction professionnelle.

Comme on peut le constater, il est intéressant et d'importance capitale, dans toute approche pédagogique, de chercher à connaître l'apprenant, que ce soit en imposant des critères d'admission aux programmes ou en cherchant à déterminer les besoins d'apprentissage de chacun, besoins qui seront comblés grâce à des mesures pédagogiques déterminées à la lumière de ce que l'apprenant sait et de ce qu'il peut faire. Ci-après, les divers aspects du savoir et du savoir-faire de l'apprenant sont présentés.

Nonobstant les courants pédagogiques, il est important, pour garantir la réussite de toute stratégie d'apprentissage, de mettre au jour ce que l'apprenant sait déjà, comme nous l'avons déjà indiqué, ne serait-ce que pour limiter l'admission aux programmes de formation.

Les critères d'admission et les examens d'admission semblent être encore les moyens privilégiés par les universités canadiennes pour chercher à mesurer le savoir et le savoir-faire acquis des futurs apprenants en traduction. Il existe cependant d'autres façons de sonder le capital cognitif des nouveaux apprenants, comme l'entrevue menée par les formateurs. Cette entrevue peut n'être qu'un moyen de déterminer si un apprenant potentiel répond ou non aux critères que l'université se sera donnés préalablement; cependant, elle peut aussi permettre de mieux connaître les apprenants et, du même coup, leurs besoins de formation. Les universités canadiennes n'utilisent pas l'entrevue comme étape à l'admission aux programmes de traduction; 
en revanche, les futurs étudiants doivent répondre à des critères d'admission et, dans $90 \%$ des universités qui offrent des programmes de baccalauréat en traduction professionnelle (Fiola 2002), se soumettre à un examen d'admission.

Pour bien comprendre ce pôle de la situation didactique que constituent les apprenants, il faut également chercher à comprendre leur motivation et chercher à savoir pourquoi ils veulent s'inscrire à un programme de traduction professionnelle.

Étant donné la nature essentiellement professionnelle des programmes de formation en traduction, on peut supposer que la plupart des apprenants veulent étudier la traduction en vue de joindre les rangs des langagiers professionnels. Sachant quelles sont les compétences requises par le marché de la traduction, il est possible de déterminer les apprentissages qui, une fois conjugués aux connaissances préalables des apprenants, permettront aux nouveaux professionnels de s'intégrer au marché de la traduction. Il est donc essentiel que le didacticien de la traduction comprenne le marché vers lequel se destinent les apprenants. Il doit également ne pas perdre de vue que si les apprenants désirent intégrer le marché du travail, c'est pour y faire carrière. Cela signifie que les programmes de traduction doivent former non seulement pour le marché actuel, mais aussi pour le marché de la traduction tel qu'il sera dans 20, 30 ou 40 ans. Malheureusement, aucune analyse du marché contemporain ne permettra de prévoir avec exactitude ce que sera le marché de la traduction dans 25 ans. En effet, il y a à peine 20 ans, l'ordinateur personnel était encore un objet de curiosité dont bien peu de traducteurs savaient se servir. À cette époque, personne n'aurait pu prévoir l'effet que l'avènement de l'informatique allait avoir sur le travail du traducteur du début du xxi ${ }^{\mathrm{e}}$ siècle, tant sur la façon de traduire que sur les communications en général.

Il est donc essentiel que les universités tiennent compte de l'évolution du marché, mais elles doivent se garder de surcharger leurs programmes pour répondre aux besoins immédiats du marché, ce qui fait dire à Yves Gambier que: «les apprenants d'aujourd'hui doivent se qualifier non pour répondre aux besoins des années $1980 \ldots$ ou 2000 mais pour faire face aux nécessités des années à venir (jusqu'en 2020-2030), pour s'adapter aux mutations prochaines - sinon même pour conduire ces mutations, pour prendre des décisions, en comprenant mieux les enjeux et implications » (2001: 74).

Il ne faut pas confondre "besoins de formation» et «besoins du marché ». Même si les motivations des apprenants sont d'ordre professionnel et économique, leurs besoins de formation sont de l'ordre des compétences et des connaissances; les besoins du marché, eux, cadrent avec des impératifs d'ordre économique. Cela ne signifie pas pour autant que les besoins des apprenants et ceux du marché sont contradictoires, mais plutôt que les universités doivent décider de la pondération des besoins de chaque partie dans la conception des programmes d'études en faisant toujours primer ceux de l'apprenant. En effet, les universités doivent tenir compte des exigences, donc des besoins, du marché de la traduction, car une bonne connaissance du marché favorisera l'intégration des apprenants au marché du travail. Il serait cependant illusoire de croire que les universités peuvent répondre aux besoins d'un marché en pleine croissance et en constante évolution. À titre d'exemple, les programmes de baccalauréat en traduction durent entre trois et quatre ans. Par conséquent, même si un programme de formation était en prise directe sur les besoins du marché, il 
pourrait au mieux, une fois les besoins ponctuels déterminés, accuser un retard minimal de trois années sur les besoins du marché.

Il parait donc judicieux de préparer les traducteurs à s'adapter aux changements du marché et leur faire acquérir des compétences dites transférables, c'est-à-dire qui leur permettront de s'accorder sur l'évolution de la profession.

Une fois que le didacticien de la traduction a cerné les connaissances préalables des apprenants potentiels, qu'il a bien compris les aspirations de ceux-ci et qu'il connaît bien le marché qui les attend, il peut déterminer leurs besoins. Ces besoins représentent ce que les apprenants devront acquérir dans le cadre de leur formation, avant de pouvoir prétendre à un poste de traducteur. Il restera donc aux concepteurs de programmes de décider ce qui en fera partie et ce qui devra être acquis par les apprenants en amont ou en aval de la formation, voire parallèlement à celle-ci.

À l'égard de la situation didactique, la notion de durée vise tant les programmes d'études que les cours et les leçons. La durée du programme dépendra bien entendu de la capacité des universités à gérer ces programmes et répondra aux motivations des apprenants. En effet, toujours à l'égard de la traduction, si les apprenants désirent intégrer le marché du travail et qu'il suffit d'un diplôme d'études de premier cycle pour y parvenir, il serait difficile de les convaincre de poursuivre des études supérieures. En revanche, si le marché du travail exigeait au minimum un diplôme d'études supérieures, rares seraient les apprenants qui se contenteraient d'une formation de premier cycle.

La durée, la fréquence et l'enchaînement des périodes de cours peut également avoir un effet sur la formation des traducteurs ${ }^{3}$. Généralement, les programmes de formation sont conçus de telle sorte que la plupart des apprenants commencent leur programme à l'automne. Toutefois, de plus en plus d'universités ont maintenant pour politique d'admettre de nouveaux étudiants au trimestre d'hiver, ce qui peut poser un problème d'enchaînement de cours. En effet, s'il est possible d'admettre des étudiants au trimestre d'hiver, il est difficile de veiller à ce que les cours proposés alors s'inscrivent dans le prolongement des cours du trimestre d'automne, à moins d'offrir tous les cours chaque trimestre, ce qui n'est pas sans alourdir le programme et la tâche d'enseignement. En revanche, si l'université admet des étudiants aux deux trimestres, elle doit pouvoir leur offrir la possibilité de poursuivre leur cheminement de manière normale. C'est alors toute la question de la structuration des apprentissages qui est remise en cause.

\subsection{L'espace}

En plus des salles de cours à proprement parler, l'élément «espace» de la situation didactique comprend tout ce qui entoure la notion d'installations et d'équipement servant à l'enseignement et à l'apprentissage, allant de l'emplacement géographique de l'université aux moyens matériels utilisés. Autrement dit, si l'on détermine, par exemple, que les apprenants doivent, à leur sortie de l'université, pouvoir utiliser une mémoire de traduction, il faut posséder les installations et l'équipement nécessaires à ce genre de formation.

À l'égard de l'espace, il faut tenir compte du nombre d'étudiants qui constitueront chaque classe, ce qui aura un effet considérable sur les mesures pédagogiques mises en ouvre dans le cadre de chacun des cours. Par exemple, pour une classe comptant 
un nombre important d'étudiants, il sera difficile, voire impossible, de recourir à un processus pédagogique axé sur l'apprentissage ou sur la formation, et la formule pédagogique axée sur l'enseignement, l'exposé magistral, sera celle que l'on privilégiera d'emblée.

On sait que dans le cadre de programmes de formation professionnelle comme ceux de traduction, la formation pratique occupe une place importante. Une des manières d'apprendre à traduire consiste à résoudre des problèmes de traduction, à faire de la traduction. Or, plus le nombre d'étudiants est grand, plus il est difficile pour les formateurs de donner aux apprenants des travaux pratiques, vu la charge de travail que représente l'encadrement que cela implique. Ce problème intéresse davantage la pédagogie que la didactique à proprement parler, mais il est important de souligner l'interdépendance de tous les éléments de la situation pédagogique, de même que de la planification didactique et pédagogique.

On pourrait longuement épiloguer sur les inconvénients des groupes comptant un nombre élevé d'étudiants. Toutefois, sans l'encadrement étroit favorisé au sein des petits groupes, l'apprenant qui en a la capacité est alors amené à prendre en main son apprentissage, ce qui favorise son autonomie, qualité appréciée par les employeurs de traducteurs. En revanche, dans un groupe réduit, le formateur est en mesure d'encadrer étroitement chaque apprenant, ce qui est tout aussi souhaitable.

Il est également possible d'inclure dans la notion d'espace celle de salle de cours. Lorsqu'on parle de salle de cours, on pense immédiatement aux dimensions, de la très grande salle à la salle très petite, ce qui sous-entend la notion de proximité du formateur et des apprenants. Plus la salle est petite, meilleures sont les chances de recourir à des stratégies pédagogiques adaptées aux besoins de chacun, ce qui, en outre, laisse entrevoir la possibilité de fonctionner à l'intérieur de groupes relativement hétérogènes. Si la salle est très grande, en revanche, le choix d'approches sera limité, et il faudra viser la plus grande homogénéité des groupes possible.

La notion d'espace appelle également à se pencher sur un phénomène nouveau: celui de la salle de cours virtuelle. Le télé-enseignement est présent dans les universités depuis longtemps mais, depuis quelques années, avec l'avènement d'Internet, un nombre croissant d'universités se tournent vers cette avenue, et ce phénomène n'est pas sans soulever un certain nombre de questions d'ordre didactique, notamment pour les programmes de formation pratique.

On peut facilement imaginer comment il est possible de transmettre un savoir, voire un savoir-faire, dans une classe virtuelle. Toutefois, il peut paraître difficile d'imaginer comment on pourrait faire acquérir aux apprenants un savoir-être en particulier, par exemple celui du traducteur.

Dans son Rapport, le Comité sectoriel indique la liste des compétences requises du jeune traducteur. Outre les connaissances et les compétences purement traductionnelles, le Rapport indique que le traducteur doit posséder d'autres compétences, celles-là d'ordre personnel, comme: «[Ê] tre doté d'une solide culture, d'une grande curiosité intellectuelle. Avoir la capacité de travailler en équipe et posséder les qualités suivantes: polyvalence, disponibilité, motivation, jugement, communication et efficacité. Le souci du client et la souplesse sont également des éléments importants » (1999: 97).

Si l'on peut s'imaginer sans trop de difficultés des mesures pédagogiques visant à inculquer aux jeunes traducteurs les notions d'efficacité et de souplesse, il n'est pas 
aussi facile de concevoir comment on peut leur faire acquérir par Internet des éléments du savoir-être nécessaires au traducteur, comme le jugement, la capacité de travailler en équipe et la motivation.

Kiraly (2000: 13) distingue le savoir-être et le savoir-faire du traducteur (translator competenceltranslation competence). Selon l'approche socioconstructiviste que préconise Kiraly, le savoir-faire et le savoir-être du traducteur ne peuvent s'acquérir que dans un contexte social. Dans cette optique, il risque d'être difficile de concilier apprentissage de la traduction et formation à distance, voire toute forme d'apprentissage. Cependant, avec la technologie de pointe permettant les échanges électroniques en temps réel, les salles de clavardage et les communications par vidéoconférence sur Internet, il est sans doute possible de recréer par des moyens électroniques une situation représentant ce qui se passe dans une salle de cours réelle, ou d'encourager les apprenants à contextualiser leur apprentissage autrement que par les contacts entre apprenants à l'intérieur d'une même salle de cours réelle.

Le recours au télé-enseignement ouvre la porte à une plus grande hétérogénéité des groupes, ce qui nécessite une diversité accrue des mesures pédagogiques mises en œuvre. Ainsi, il faudrait se garder de croire que le télé-enseignement permettra de multiplier le nombre d'étudiants inscrits aux programmes car, d'un point de vue pédagogique, l'hétérogénéité des classes, réelles ou virtuelles, appelle une pédagogie adaptée, donc un encadrement plus étroit que dans les classes homogènes.

\subsection{Les formateurs}

Quatrième et dernier élément, les formateurs ont une influence considérable sur la situation didactique, et l'influence qu'ils ont sur les programmes dépend de leurs intérêts, de leur statut professoral, de leur formation pédagogique et de leurs motivations.

Il convient dès le départ de faire une distinction entre deux catégories de formateurs. Au Canada, la formation des traducteurs est confiée à la fois à des professeurschercheurs, qui occupent un poste à l'université, et à des chargés de cours. Les chargés de cours sont généralement des professionnels de la traduction à qui l'on confie un cours. Ces chargés de cours sont choisis en fonction d'exigences relatives au cours qui leur est confié, à l'égard de leur formation et de leur expérience professionnelle. Si les chargés de cours sont embauchés selon les besoins ponctuels de formation, pour les professeurs-chercheurs en poste à l'université, l'enseignement ne constitue qu'une partie de leur tâche de travail. La motivation à enseigner n'est donc pas la même chez les professeurs et les chargés de cours. Les intérêts des formateurs peuvent également influer sur la composition des programmes et des cours.

Pour les professeurs de traduction, l'enseignement est un élément de leur tâche de travail, au même titre que la recherche et la supervision d'étudiants aux cycles supérieurs, par exemple, et la traduction est pour eux un objet d'étude, non une profession. En revanche, les chargés de cours sont recrutés précisément pour leur savoir-faire dans un domaine en particulier. Toutefois, en raison de leurs préoccupations professionnelles et dû au fait qu'ils sont relativement détachés des préoccupations de gestion universitaire, les chargés de cours ne bénéficient pas toujours de la vue d'ensemble nécessaire à la cohésion de la formation, notamment en ce qui concerne les mouvances théoriques sous-jacentes aux programmes. D'où, on peut se demander s'il serait préférable de confier certains cours à l'un ou à l'autre groupe de formateurs. 
L'enseignement de la traduction au niveau universitaire est empreint d'un certain paradoxe. D'un côté, les professeurs tentent de convaincre tous et chacun qu'il ne suffit pas d'être bilingue pour être traducteur professionnel. D'un autre côté, il semble évident qu'il suffit d'être allé à l'université jusqu'à la maitrise, voire jusqu'au doctorat, pour pouvoir enseigner. Le formateur doit être langagier, ou traductologue, cela va de soi, mais ne devrait-il pas également posséder une certaine compétence pédagogique pour faire valoir sa connaissance de la compétence traductionnelle? Certes, ce phénomène n'est pas exclusif à la traduction, car il est répandu à tout le milieu universitaire, à l'exception du domaine des sciences de l'éducation, où la pédagogie et la didactique sont au centre des préoccupations. Toutefois, le maintien du statu quo ne se justifie pas. En milieu universitaire, la compétence pédagogique est une compétence attendue, c'est-à-dire qu'il n'est pas nécessaire d'en faire la preuve avant de se voir confier un premier groupe d'étudiants. Cependant, des chercheurs comme Birgitta Englund Dimitrova, Daniel Gouadec, Don Kiraly et Anthony Pym (Pym s.d.: 2) tentent actuellement de jeter les assises d'une approche raisonnée de l'enseignement de la traduction, respectivement en Suède, en France, aux États-Unis et en Espagne. Au Canada, l'Université d'Ottawa offre, dans le cadre de son programme de maîtrise en traduction, un séminaire intitulé «Pédagogie de la traduction ». Cependant, les étudiants inscrits au programme ne sont pas tenus de suivre ce séminaire.

Tout comme il est nécessaire et utile de comprendre les motivations des étudiants, il l'est tout autant de comprendre les motivations des formateurs. Comme exemples de motivation, citons le goût de partager son expérience, l'envie d'assurer une relève compétente pour la profession, la capacité d'enseigner dans un domaine plutôt que dans un autre, ou l'obligation purement terre-à-terre de s'acquitter de sa tâche d'enseignement. Peu importe les motivations, elles auront un effet sur l'enseignement prodigué et, par conséquent, sur la qualité de la formation.

\section{Interdépendance des quatre éléments}

Tous ces éléments sont interdépendants, solidaires. Afin d'illustrer ce principe, reprenons les critiques contenues dans le Rapport du Comité sectoriel (1999: 32) à l'endroit des programmes de formation. Les nouveaux diplômés accuseraient les faiblesses suivantes :

- formation générale trop limitée;

- formation pratique lacunaire (formation trop théorique);

- difficulté à travailler de façon autonome.

Les universités qui souhaitent répondre aux critiques du Rapport pourraient modifier la formation qu'elles dispensent, notamment en approfondissant la formation générale des apprenants, en axant la formation sur le contenu pratique et en favorisant le travail autonome. Voyons maintenant comment tout exercice de modification d'un programme d'étude mobilise les éléments de la situation didactique.

À leur arrivée à l'université, les apprenants en traduction - et c'est sans doute le cas de la majorité des apprenants de même niveau - accuseraient une faiblesse à l'égard des connaissances générales. Si l'université décidait d'ajouter des cours de formation générale à ses programmes, cela ne serait pas sans avoir de conséquences sur la composition du corps professoral, sur la composition du corps étudiant et sur 
la durée des programmes. En effet, il faudrait soit prolonger les programmes, soit retirer des cours pour faire la place à ce nouvel aspect de la formation. Si l'on décide de maintenir la durée des programmes en retranchant certains éléments de la formation pour y inclure la formation en culture générale, il faudrait recruter parmi la clientèle étudiante potentielle les apprenants qui possèdent déjà les éléments de formation qui ont été retranchés des programmes mais qui sont toujours considérés comme essentiels à la compétence traductionnelle. D'une manière ou d'une autre, ce changement aura des répercussions sur la composition du corps professoral, étant donné qu'il faudra recruter des formateurs dans de nouveaux domaines. Comme on peut le constater, tous les éléments de la situation didactique sont solidaires.

On critique également les programmes de formation en disant qu'ils sont trop théoriques, pas suffisamment pratiques. Toutefois, un programme d'études qui serait essentiellement pratique n'aurait pas sa place à l'université. En effet, pourquoi entreprendre une formation à l'université si cette formation consiste essentiellement à faire de la pratique, chose que l'on peut très bien faire ailleurs qu'en milieu universitaire, éventuellement contre rémunération? On peut également se demander si le milieu universitaire est véritablement le meilleur endroit pour assurer la formation pratique des jeunes traducteurs. Il parait évident que les praticiens seraient beaucoup mieux armés que les universitaires pour assurer ce genre de formation. Toutefois, théorie et pratique vont de pair, et c'est notamment pour cette raison que l'enseignement de la traduction a sa place à l'université. Comme le souligne Neubert (1989), sans pratique, la théorie est stérile, mais sans théorie, la pratique est aveugle. Pour favoriser le développement de l'autonomie des étudiants, il faut les amener à pouvoir agir seuls, certes, par une formation pratique, mais aussi à pouvoir raisonner sur la portée de leurs actes, grâce à une solide base théorique. Bien entendu, il ne fait aucun doute que l'autonomie peut se manifester à divers degrés selon les personnes, mais il reste à savoir s'il est possible de développer cette capacité d'autonomie dans le cadre d'une formation universitaire. Une façon d'attaquer le problème serait d'augmenter le sens des responsabilités des apprenants, notamment en leur faisant jouer un rôle de premier plan dans leur formation. Toutefois, pour y arriver, il faut procéder à un véritable changement de paradigme et considérer la pédagogie de la traduction comme un processus d'apprentissage, non d'enseignement, processus par lequel l'apprenant est au cœur des mesures pédagogiques et où le formateur joue un rôle d'accompagnateur. L'apprenant se sentirait ainsi aux commandes de son apprentissage.

\section{Conclusion}

Il fait peu de doute que la formation du traducteur doit maintenant être un alliage de pratique et de théorie, dont les dosages respectifs restent encore à déterminer. Parce qu'un programme d'étude est un ensemble intégré de mesures visant à la formation d'apprenants, il faut que les mesures pédagogiques et didactiques mises en œuvre soient intégrées aux autres éléments de la situation didactique. C'est pourquoi il paraît difficile, voire illusoire, comme l'écrit Valentine (1998:5), de chercher à créer un modèle de formation unique; il faut plutôt chercher à analyser chaque élément de la situation didactique pour créer des programmes véritablement intégrés au contexte. 
Par conséquent, si théorie et pratique de la traduction doivent aller de pair, il en est de même de l'enseignement, de la formation et de l'apprentissage, qu'il faut éclairer dans la pratique d'un éclairage théorique. Sur ce point, il paraît nécessaire de chercher à établir les fondements d'une didactique propre à la traduction professionnelle.

\section{NOTES}

1. Le terme "programme d'études» désigne ici un ensemble intégré de mesures visant au développement des savoirs, des savoir-faire et des savoir-être nécessaires pour qu'un apprenant soit compétent en traduction professionnelle.

2. Comité composé de représentants du milieu universitaire, des secteurs public et privé, des associations professionnelles et des fabricants de logiciels et de matériel informatique.

3. Dans les universités offrant des programmes de traduction au Canada, les périodes de cours sont généralement de trois heures par semaine, s'échelonnant sur les quinze semaines que dure chacun des trimestres. Les cours sont concentrés dans les trimestres d'automne et d'hiver, mais des universités offrent également des cours pendant le trimestre de printemps.

\section{RÉFÉRENCES}

CARd, C. (1990) : «Does university training measure up?», dans M. C. Cormier (dir.), La traduction au Canada. Les acquis et les défis. Actes $d u 2^{e}$ Congrès $d u$ Conseil des traducteurs et interprètes du Canada tenu à Montréal les 31 mai, $1^{\text {er }}$ et 2 juin 1990, Ottawa, Conseil des traducteurs et interprètes du Canada, p. 53-60.

Chaduc, M.-T. et al. (1999): Les grandes notions de pédagogie, Formation des enseignants, Enseigner, Paris, Armand Colin/Bordas/HER, 332 p.

Comité Sectoriel de L'industrie CANAdienne de la traduction (1999): L'industrie canadienne de la traduction - Stratégie de développement des ressources humaines et d'exportation - Rapport final, Ottawa, Industrie Canada, $105+\mathrm{x}$ pages.

Delisle, J. (1980): L'analyse du discours comme méthode de traduction, Ottawa, Les Presses de l'Université d'Ottawa, $282 \mathrm{p}$.

Delisle, J. (1987): La traduction au Canada/Translation in Canada 1534-1984, Ottawa, Conseil des traducteurs et interprètes du Canada/Les Presses de l'Université d'Ottawa, 436 p.

Fiola, M. A. (2002): «Aptitudes, compétences et critères d'admission aux programmes de traduction» dans Actes du Congrès de la Fédération internationale des traducteurs 2002, La traduction: des idées nouvelles pour un siècle nouveau, Vancouver, 5 pages.

GAmbier, Y. (2001) : «Professionnaliser la formation des traducteurs?», p. 71-76, dans D. GouAdeC (dir.), Formation des traducteurs (2), actes du Colloque international Rennes 22-23 septembre 2000, Paris, La maison du dictionnaire.

Goffin, R. (1971): «Pour une formation universitaire 'sui generis' du traducteur», dans Meta, $16-1 / 2$, p. $57-68$.

Horguelin, P. A. (1975): «Table ronde sur l'enseignement de la traduction », dans Meta, 20-1, p. $42-57$.

Kiraly, D. (2000): A Social Constructivist Approach to Translator Education: Empowerment from Theory to Practice, Manchester (Royaume Uni)/Northampton (Massachusetts), St. Jerome Publishing, $207 \mathrm{p}$.

Lavault, É. (1998): Fonctions de la traduction en didactique des langues: Apprendre une langue en apprenant à traduire, nouvelle édition, Paris, Collection «Traductologie», Didier Érudition, $126 \mathrm{p}$.

Neubert, A. (1989): “Translation as Mediation” dans R. Kölmel et J. Payne (dir.), Babel: The Cultural and Linguistic Barriers Between Nations, Aberdeen, Aberdeen University Press, viII +188 p. 
Pyм, A. (s. d.): Trial, Error and Experimentation in the Training of Translation Teachers [En ligne], $<$ http://www.fut.es/ apym/on-line/trialanderror.pdf>, page consultée le 5 juin 2002, 13 p.

Roberts, R. P. (1985): «University Education and Professional Skills», dans Proceedings of the National Symposium on Linguistic Services held in Ottawa, October 9-12, 1984, Ottawa, Secretary of State, p. 343-352.

Ulrych, M. (1995) : «Real-world criteria in translation pedagogy», dans C. Dollerup et V. Appel (dir.), Teaching Translation and Interpreting 3, Amsterdam/Philadelphie, John Benjamins Publishing Company, p. 251-259.

Valentine, E. (1998): «Perspectives d'optimisation de la formation du traducteur: quelques réflexions», dans Z. Guével et E. Valentine (dir.), Traduction et langues de spécialité, Approches théoriques et considérations pédagogiques, Québec, Centre international de recherche en aménagement linguistique, Faculté des lettres, Université Laval, p. 3-23.

Vinay, J.-P. et J. Darbelnet (1958): Stylistique comparée du français et de l'anglais: Méthode de traduction, Bibliothèque de stylistique comparée, Laval, Éditions Beauchemin, 332 p. 